\title{
PROKLA-Redaktion
}

\section{Editorial: Ist der entfesselte Weltmarkt noch zu bändigen?}

I.

Auf den ersten Blick scheinen sich die Diskurse zu ähneln: Um die Wende zum 20. Jh. wurde die „Anarchie des Marktes“ für die heftig wütenden ökonomischen Krisen der Vergangenheit verantwortlich gemacht. Sie verunsicherten mit sozialstaatlich nicht abgesicherter Arbeitslosigkeit und daraus resultierendem Massenelend, Firmenzusammenbrüchen und sozialen Unruhen die kapitalistischen Industriegesellschaften. Von der „Organisation des Kapitalismus“, von der Einführung regulierender Elemente in die Marktwirtschaft gegen die ungezügelte Konkurrenz haben sich sogar liberale Wirtschaftstheoretiker wie Naumann und erst recht Sozialdemokraten wie Hilferding und andere „Austromarxisten“, später auch Kommunisten eine Verhinderung der Krisen oder zumindest eine Linderung ihrer schlimmsten Auswirkungen versprochen. Bis zur großen Depression der frühen dreißiger Jahre war diese Sichtweise weit verbreitet: Die regulierende Organisation des Kapitalismus könne dazu beitragen, Krisen zu vermeiden. Weil man diese einfache Lehre in den „roaring twenties“ nicht befolgt habe, sei es zu der großen Weltwirtschaftskrise nach dem Schwarzen Freitag vom Oktober 1929 gekommen. So nahezu einvernehmlich die Krisentheoretiker der 20er und 30er Jahre.

Hundert Jahre später, am nächsten „fin de siècle" sind wir Zeugen eines durchaus vergleichbaren Diskurses: Man müsse das entfesselte, weil fast vollständig deregulierte globale Finanzsystem bändigen, um drohenden Finanzkrisen vorzubeugen und, wenn sie denn eingetreten seien, politische Lösungspakete gegen ihre Konsequenzen zu schnüren. Die anarchischen Märkte sollen wieder organisiert werden wie hundert Jahre zuvor, nur die Sprache hat sich geändert. Heute heißt die Organisation des Kapitalismus: Re-Regulierung des globalen Finanzsystems. Die in der wissenschaftlichen und politischen Debatte ventilierten Vorschläge reichen von Regeln zur Herstellung einer erhöhten Transparenz der Märkte, einem verbesserten Risikomanagement der Banken unter der Aufsicht von privaten Rating-Agencies, verschärften Vorschriften hinsichtlich der Eigenkapitalausstattung, Kontrollen besonders volatiler Investment-Fonds und der bislang ohne wirksame Bankenaufsicht operierenden freien Bankzonen bis zur Besteuerung kurzfristiger Kapitalbewegungen, einer Mitverantwortung von Gläubigern (und nicht nur von Schuldnern) im Falle akuter Finanzkrisen und Kapitalverkehrskontrollen an den Grenzen von nationalstaatlichen Währungsgebieten. Auch eine Reform der Institutionen der Weltwirtschaft, von Weltbank und Internationalem Währungsfonds bis zur Welthandelsorganisation, ist im Gespräch (vgl. dazu die Beiträge von Heribert Dieter und Susanne Lütz). Die Schuldenkrise der Dritten Welt in den achtziger Jahren und die schweren Finanzkrisen der neunziger 
Jahre in Asien, in den Transformationsländern Osteuropas und in Lateinamerika bieten genügend Belege für die Zerstörungskraft des Geldes, wenn es denn in eine (globale) Krise gerät.

II.

Der Therapievorschlag "Organisation“ bzw. Re-Regulierung" des Kapitalismus kann aber nicht so recht überzeugen, weil die zugrundeliegende Diagnose fragwürdig ist. Die Anarchie des Marktes mag zwar Disproportionen (Überproduktion an einer Stelle, Unterproduktion an einer anderen) erklären, nicht aber allgemeine und periodisch wiederkehrende Krisen. Bereits im frühen 19. Jh. machte Sismondi die Unterkonsumtion der Massen für die Krisen verantwortlich: sie führe zu Nachfragemangel mit Produktionsausfällen und Arbeitslosigkeit. Verschiedene Varianten dieser „Unterkonsumtionstheorie“ sind bis heute bei vielen kritischen Ökonomen, marxistischen Sozialwissenschaftlern und sozialdemokratischen Theoretikern verbreitet. Allerdings ist auch diese Krisenerklärung nicht befriedigend, denn die Massenkaufkraft, die aus den Lohneinkommen resultiert, ist stets kleiner als der Wert des Sozialprodukts - sonst könnte es keinen Profit geben. Ob es tatsächlich zu einem „Nachfragemangel“ und einer Krise kommt, hängt jedoch von zwei weiteren Faktoren ab. Erstens wie sich die Konsumnachfrage der Bezieher arbeitslosen Einkommens entwickelt und, was wichtiger ist, wie hoch die Investitionsnachfrage ist, die dem Verwertungskalkül der Investoren in Produktivkapital und deren Gläubiger unterliegt.

Zur Krisenerklärung sind wir damit auf die komplexen Bestimmungsgründe der Dynamik des kapitalistischen Akkumulationsprozesses verwiesen. Während die moderne Neoklassik in ihrer Fixierung auf statische Gleichgewichtskonstellationen dynamische Prozesse weitgehend ausblendet und daher Krisen immer nur als punktuelle und im Prinzip vermeidbare Störungen eines an sich harmonischen Ganzen auffasst, stellte Marx die krisenhafte Dynamik kapitalistischer Akkumulation ins Zentrum seiner Analyse. Die formelle Möglichkeit der Krise ist bereits mit der Form von Ware und Geld gegeben: im Unterschied zum bloßen Tausch von Produkten können Kauf und Verkauf auseinanderfallen, der gesellschaftliche Reproduktionszusammenhang also zerreiBen, weil die einen verkaufen und das Geld nicht wieder ausgeben und die anderen verkaufen möchten, aber keine Käufer finden. Dass diese bloße Möglichkeit der Krise unter kapitalistischen Verhältnissen Wirklichkeit wird, wollte Marx mit seiner Krisentheorie zeigen. Während die kapitalistische Produktion einerseits zu beständiger Ausdehnung tendiert (durch Steigerung der Produktivität und um Skaleneffekte zu nutzen), findet die Konsumtion immer wieder immanente Grenzen. Beschränkt ist nicht nur die Konsumtion der breiten Bevölkerung, sondern, so Marx, auch die „produktive Konsumtion“, d.h. die Investitionsnachfrage der Kapitalisten. Für letztere ist bei Marx die Profitrate und deren Aufteilung in Zinsen und Gewinne entscheidend. Die Profitrate hängt von einer Vielzahl von Faktoren wie der Lohnentwicklung und den Kosten für neue Technologien ab, die Entwicklung der finanziellen Rendite von Angebot und Nachfrage auf Finanzmärkten und der Zusammenstellung von „Portefeuilles“.

Dass die Investitionsnachfrage der Kapitalisten nicht ausreicht, um Vollbeschäftigung herzustellen, dass es immer wieder zur Überakkumulation von Kapital und zu Krisen kommt, ist nach Marx kein unglücklicher Betriebsunfall des Kapitalis- 
mus, sondern eine Konsequenz seines normalen Funktionierens. So schlimm sich diese Krisen auch auf die Lebensverhältnisse vieler Menschen auswirken - für den Kapitalismus als System haben sie durchaus positive Wirkungen: Vernichtung und Entwertung von Kapital beseitigen die Überakkummulation; Arbeitslosigkeit und Lohnsenkungen verbessern die Verwertungsbedingungen, so dass die Profitrate wieder steigen und ein neuer Akkumulationszyklus einsetzen kann - bis auch dieser wieder in einer Krise endet.

Die Bedeutung, die das Verhältnis von Profiterwartungen und Zinssatz für die Dynamik der Investitionen und den Umfang der Beschäftigung hat, wurde im 20. Jh. besonders von Keynes hervorgehoben, der wie Marx von der endogenen Krisenhaftigkeit des Kapitalismus ausgeht. Keynes hat aus seiner Analyse durchaus radikale Konsequenzen gezogen. Zwar hat Keynes ein kooperatives weltwirtschaftliches Währungs- und Finanzregime einschließlich Begrenzungen beim internationalen Kapitalverkehr als beste Lösung vorgeschlagen, jedoch forderte er in zugespitzten Situationen noch weitaus stärkere staatliche Eingriffe. Nicht nur sollte die Freiheit des Kapitalverkehrs an den nationalstaatlichen Grenzen enden - gemäß seiner These, dass die Freiheit des Warenhandels gut, die Freiheit des transnationalen Kapitalverkehrs aber schlecht für den Wohlstand der Nationen seien. Vor allem sollte die Zentralbank für niedrige Zinsen sorgen, um eine für Vollbeschäftigung ausreichende Rentabilität der Investitionen zu gewährleisten. Für dieses Ziel wollte Keynes auch den „sanften Tod des Rentners" (derjenigen, die von Finanzrenditen leben) in Kauf nehmen: die Dominanz der Geldvermögensbesitzer über die realen Produktivvermögen sollte gebrochen werden. Falls dies nicht ausreichen sollte, schloss Keynes auch eine „Sozialisierung der Investitionen “ nicht aus.

In Zeiten der Globalisierung haben sich die Bedingungen jedoch grundlegend geändert. Spätestens seit Mitte der siebziger Jahre ist in einem Prozess furioser Deregulierung fast jede Schranke der Mobilität von Geldvermögen und Geldkapital geschliffen worden. Und da die politisch gesetzten Grenzen der freien Mobilität von Kapital kaum noch existieren, haben die Finanzinstitutionen auf den globalisierten Finanzmärkten das Ihre dazu beigetragen, um die Kapitalbewegungen nun tatsächlich in einem Ausmaß zu beschleunigen, dass nachgerade just-in-time der Vergleich von Renditen von Investitionen im realen Sektor der Ökonomie und Finanzanlagen an irgendeinem Punkt der Erde möglich geworden ist.

Die beschleunigte Globalisierung ökonomischer Prozesse seit den siebziger Jahren findet ihren Kern in der Liberalisierung des internationalen Kapitalverkehrs. Zwar zeigen sich auch deutliche Tendenzen bei der Integration beim Handel von Gütern und Dienstleistungen, jedoch sind diese Tendenzen eher langfristig feststellbar. Im Vergleich etwa zur ebenfalls globalisierten Zeit vor dem Ersten Weltkrieg sind die Arbeitsmärkte eher abgeschottet. Im Rahmen der Liberalisierung des internationalen Kapitalverkehrs entwickelten sich eine ganze Reihe neuer Finanzintermediäre und Anlagestrategien. Seit den achtziger Jahren schießen Investment-, Hedge- und andere Fonds wie Pilze aus dem Boden. Sie sammeln Geldvermögen ein und legen es zur höchstmöglichen Rendite ihrer Klientel weltweit an. Die Anlage mag in realen Objekten und Projekten erfolgen. Doch ist sie in der Regel so kurzfristig und ,volatil“, d.h. immer auf dem Absprung, dass langfristig angelegte Projekte der Entwicklung auf diese Weise kaum zu 
finanzieren sind. Es zählt der „shareholder value“, der kurzfristige Erfolg - gemessen an den auf globalen Finanzmärkten erzielbaren Renditen.

Die modernen Globalisierungsprozesse wurden möglich durch den Rückgriff auf die fossilen Energieträger und die Entwicklung passender Energiewandlungssysteme in Produktion, Transport, Distribution; durch systematische, d.h. kapitalistische Produktion von Überschüssen; durch die Entwicklung ausgreifender und inzwischen weltumspannender Kommunikationsnetze (Internet) auf der Grundlage der modernen Informationstechnologien; durch eine Reduzierung der Transaktionskosten; durch die Herausbildung globaler Standards, getragen von den RatingAgencies bis zu den ISO-Normen, von den global operierenden Anwaltskanzleien bis zu den globalen Beherbergungs- und Ernährungsketten; durch die Entwicklung global operierender Akteure, insbesondere von Finanzinstitutionen. Letztere haben mit „Finanzinnovationen“ dafür gesorgt, dass sich das Karussell der globalen Finanzmärkte immer schneller zu drehen vermag - und dabei können dessen Mitfahrer ins Schleudern geraten. Die Globalisierung ist also wesentlich eine Beschleunigung in der Zeit und eine Expansion im Raum mit der Folge, daß Distanzen reduziert werden. Natürliche und politische Grenzen der Konkurrenz auf Waren-, Kapital-, Finanz- und Währungsmärkten sind weitgehend verschwunden und mit ihnen die Möglichkeit, an den Grenzen regulierend (durch Kapitalverkehrskontrollen; Konvertibilitätsschranken, Zölle etc.) einzugreifen. Dies ist ein Grund dafür, daß „Re-Regulierung“ meist als „Entschleunigung“ und „Re-Regionalisierung" konzipiert wird.
III.

In den Finanzkrisen zeigt sich schlagend, dass Geld eine soziale Beziehung zwischen Schuldnern und Gläubigern zum Ausdruck bringt. Die einen haben Forderungen $\mathrm{zu}$ bedienen und die anderen verbuchen diese Forderungen als (Geld-)Vermögenswerte, die sie als ökonomische Aktivposten in ihren ökonomischen Strategien einsetzen. Die Gläubiger können daher nicht einfach auf Forderungen verzichten, wenn Schuldner Schwierigkeiten haben, diese zu bedienen. Dies haben die Nationalstaaten und insbesondere die international operierenden Institutionen des globalen Finanzsystems (Bank für Internationalen Zahlungsausgleich, Internationaler Währungsfonds, Weltbank, Pariser und Londoner „Club“ etc.) in der Vergangenheit zur ersten Regel erhoben, um ein „Systemrisiko“, das heißt den Zusammenbruch wichtiger Gläubiger, weil diese ihr Geldvermögen abschreiben müssen, gar nicht erst eintreten zu lassen. Verschuldete Länder wurden dazu veranlasst, alles zu tun, um die Schuldendienstfähigkeit wieder herzustellen, was stets auf Kosten einer Mehrheit der Bevölkerung erfolgt. Ihr Einkommen sinkt und soziale Leistungen werden scharf beschnitten. Um Schulden bedienen zu können, wurden den Schuldnern in der Regel auch zusätzliche finanzielle Mittel zur Verfügung gestellt (Umschuldung, Schuldenrestrukturierung), mit denen aber nichts anderes erreicht werden sollte, als die soziale Geldbeziehung zwischen Gläubiger und Schuldner aufrechtzuerhalten. Diese Art von Regulierung gemäß dem so genannten „Konsens von Washington" war in den vergangenen zwei Jahrzehnten insofern erfolgreich, als Gläubigerinstitutionen und Gläubigerländer nicht gezwungen wurden, größere Vermögenswerte abzuschreiben, sondern ganz im Gegenteil erhebliche Gewinne 
verbuchen konnten. Das Systemrisiko konnte also vermieden werden. Der Preis dafür war freilich hoch, denn die Schuldner haben es nicht vermocht, ihre Schulden dauerhaft zu reduzieren oder gar auf die Seite der Gläubiger zu wechseln. Diejenigen Schuldner, von denen nicht zu erwarten ist, dass sie in absehbarer Zeit die Schuldendienstfähigkeit wiederherstellen, sind von den globalen Finanzmärkten weitgehend abgekoppelt worden. Dies trifft für einen großen Teil der afrikanischen Länder südlich der Sahara und für einige Nachfolgestaaten der Sowjetunion zu. Ihr Rating ist so schlecht, dass privates Kapital einen vorsichtigen Bogen um sie macht. Dieser Stand der Dinge sowie der Schock der asiatischen Finanzkrise haben dazu beigetragen, dass inzwischen über einen „Post-Washington-Konsens“ und neue Regeln des Krisenmanagements, in denen mehr als zuvor auf die Leistungsfähigkeit der Schuldner Rücksicht genommen wird, nachgedacht wird. Auch sollen die privaten Gläubiger nicht mehr weitgehend ungeschoren aus Finanzkrisen herauskommen, sondern einen größeren Teil der Kredite abschreiben.

Eine Folge der in der Vergangenheit aufgebauten Gläubiger-Schuldner-Beziehung auf globaler Ebene ist unvermeidlich eine wachsende soziale und ökonomische Ungleichheit in der Welt. Ein Blick in die jährlichen Berichte des UNDP (Berichte über die menschliche Entwicklung), aber auch in die Veröffentlichungen von Weltbank und Internationalem Währungsfonds zu den Konsequenzen der jüngsten Finanzkrisen, unterlegt diesen Sachverhalt mit statistischen Angaben. Freilich ist der Prozess der globalen Umverteilung zugunsten der „Reichen“ vielschichtig. Er findet innerhalb jeder Nation (bzw. innerhalb jedes Währungsraums) statt, er hat regionale Besonderheiten und er voll- zieht sich vermittelt über die Entwicklung von Zinsen und Preisen für Rohstoffe und Industrieprodukte (Terms of Trade) auf globalen Märkten. Er wird moderiert durch staatliche Hilfsleistungen (Entwicklungszusammenarbeit, technische $\mathrm{Zu}-$ sammenarbeit) und private und offentliche Transfers (Technologietransfer oder Transfers im Rahmen der Klimaabkommen). Der Prozess der Umverteilung ist turbulent und nicht ohne weitergehende Analyse auf die sozialen Disparitäten einer einfachen finanziellen Beziehung zwischen Gläubigern und Schuldnern zurückzuführen. Obendrein impliziert die monetäre Ungleichheit auch ungleiche Zugänge zu Wissen, Technik und Naturressourcen. Sie resultiert also in ungleichen Entwicklungschancen von ganzen Gesellschaften.

IV.

Eine Re-Regulierung der Finanzmärkte ist also unausweichlich, und sie wird inzwischen selbst von jenen nicht mehr gänzlich ausgeschlossen, die noch vor wenigen Jahren der totalen De-Regulierung und möglichst weitgehenden Privatisierung das Wort geredet haben. Doch reicht die ReRegulierung aus? Erinnern wir uns an die zentrale Bedingung, unter denen nach Keynes Investitionen nur durchgeführt werden. Der Vergleich der erwarteten Verwertung von Investitionen in Produktivkapital mit auf Finanzmärkten erzielbaren Zinsen muss zugunsten der Rendite von Investitionen in Produktivvermögen ausfallen. Infolge der Globalisierung der Finanzmärkte haben jedoch die geldpolitischen Institutionen, insbesondere die Zentralbanken, in den meisten Ländern der Welt die Zinssouveränität weitgehend verloren. Zwar können Zentralbanken die Zinsen anheben, um den „Standort“ für Geldvermögen attraktiv zu machen; sie werden aber Schwierigkeiten bekommen, 
Zinsen zu senken, weil dies unweigerlich „Kapitalflucht“ auslösen würde. Dies kann zwar unter besonderen historischen Bedingungen für spezifische Länder - Länder mit hohen Leistungsbilanzüberschüssen ohne Inflationsgefahren eine strategische Option sein (so wie in Japan in den neunziger Jahren) -, ist aber keine Möglichkeit für die Masse der Länder, etwa für Länder, die eine defizitäre Leistungsbilanz aufweisen und daher auf Kapitalimporte angewiesen sind oder für Länder, bei denen eine Abwertung sofort das Vertrauen in das nationale Geld zerstört. Es ist dies aber auch keine Option, die den international operierenden Investmentfonds, Versicherungen und anderen Institutionen der Finanzanlage gefallen würde. Denn dann könnten sie die Rendite für ihre Klientel nicht so steigern, wie es in den vergangenen zehn bis zwanzig Jahren geschehen ist.

Die Finanzkrisen der vergangenen Jahrzehnte sind also keineswegs nur Folge der Deregulierung der Finanzmärkte. Es wird auch das Verhältnis von realem Wachstum, Profitrate und monetären Zinsen zu berücksichtigen sein, wenn die Finanzkrisen erklärt und Maßnahmen der ReRegulierung entwickelt werden sollen. Ein Blick in die historische Statistik der OECD zeigt, dass Ende der siebziger bzw. Anfang der achtziger Jahre ein struktureller Umschwung des Verhältnisses von Profitrate und realer Wachstumsrate des BIP und den monetärer Größen, Zinsen und Renditen auf Finanzanlagen, stattgefunden hat: Während in den Industrieländern von 1960 bis etwa 1979 die realen Wachstumsraten des Bruttoinlandsprodukts oberhalb der realen langfristigen Zinssätze (Nominalzinssätze abzüglich der Inflationsrate) lagen, befinden sich seit Beginn der achtziger Jahre genau umgekehrt die langfristigen Realzinsen oberhalb der realen Wachstumsrate (eine Zusammenstellung der Daten findet sich in: Altvater/Mahnkopf, Grenzen der Globalisierung, Münster 1999: 169). Selbst sinkende Realzinsen seit der zweiten Hälfte der achtziger Jahre haben dieses Bild nicht grundlegend verändert, da auch die realen Wachstumsraten des Bruttoinlandsproduktes rückläufig gewesen sind.

Die OECD hat sich der Frage des „zu hohen“ Realzinsniveaus im Wirtschaftsausblick (Nr. 53) vom Juni 1993 angenommen. Sie nennt drei Gründe für das „seit 1980 beobachtete hohe Realzinsniveau“ (OECD 1993: 29): Erstens die Zunahme der öffentlichen Verschuldung und eine Geldpolitik, die in den siebziger Jahren die Inflation mit Hilfe hoher kurzfristiger Zinsen zu bekämpfen trachtete; zweitens hohe Zinsforderungen der „Investoren“, um das Inflationsrisiko abzufangen, und drittens die Globalisierung der Finanzmärkte, durch die „Marktmechanismen auf breiter Front stärker zum Zuge (kamen)" (OECD 1993: 30), so dass die Marktzinssätze „die Funktion der Kreditallokation übernahmen, während administrative, nicht kostenbezogene Methoden aus dieser Funktion verdrängt wurden“ (ebd.). Tendenziell sinkende reale wirtschaftliche Wachstumsraten und steigende Realzinsen öffneten eine Schere, die zur Umverteilung zu Lasten der Lohnempfänger führte und es ist fraglich, ob dieser Trend durch „Re-Regulierung“ verändert werden kann.

Noch eindeutiger als in den Industrieländern ist das Verhältnis von realer Wachstumsrate und Realzinsen in den verschuldeten Ländern der „Dritten Welt“, wo zeitweise Realzinsen in zweistelliger Größenordnung auf Außenkredite in fremder Währung zu leisten waren. Dagegen war eine Konstellation von realen Zinsen unterhalb von realer Rendite und Wachs- 
tumsrate eine Grundlage für den keynesianischen Klassenkompromiß, für die Dynamik der fordistischen Regulationsweise, für die ,golden years“ der Kapitalakkumulation und für die Dynamik des „Entwicklungsstaats“ in den Ländern der „Dritten Welt" vom Ende des zweiten Weltkriegs bis etwa zur Mitte der Siebziger Jahre.

Wenn die Zinsen dauerhaft oberhalb der realen Wachstumsrate liegen, hat dies beträchtliche negative Konsequenzen: Erstens werden bei schlagartigen Erhöhungen der Realzinsen die Schuldner systematisch überfordert; irgendwann sind sie nicht mehr in der Lage, den Schuldendienst zu begleichen. Die Schuldenkrise bricht aus. Deren „Regulation“ bestand in der Vergangenheit, wie schon ausgeführt, im wesentlichen darin, das Übergreifen der Schuldenkrise auf Gläubiger, also das „Systemrisiko“ zu verhindern. Die zweite Konsequenz zu hoher Realzinsen besteht in der Behinderung oder gar Unterdrückung der Investitionstätigkeit in Produktivvermögen. Und die dritte Konsequenz ist in vielen Fällen eine Überforderung der Schuldner. Denn der Schuldendienst wird, sofern die Realzinsen über der realen $\mathrm{Zu}$ wachsrate des Sozialprodukts liegen, aus der Substanz zu zahlen sein: Kapitalbildung findet nicht statt und selbst Ersatzinvestitionen bleiben aus. Die Infrastruktur verfällt und auch ins „Humankapital“, also in Bildung und Ausbildung, in Gesundheit und Sozuiales, wird nicht mehr investiert. So kann in dürren Worten die Situation in einer Reihe von verschuldeten und von Schulden- und Finanzkrisen gebeutelten Ökonomien in Lateinamerika, Afrika und Asien geschildert werden.

Im Zuge der finanziellen Globalisierung verändert sich also der Charakter der kapitalistischen Akkumulation. Produktives Kapital wird, soweit wie physisch und sozial möglich, finanziell mobilisiert, so dass nun Unternehmen auch von seiten ihrer Aktionäre (der „Shareholders“) unter Druck geraten, eine reale Rendite zu erwirtschaften, die an die bei Finanzanlagen erzielbare Rendite auf globalen Märkten („Benchmarking“) heranreicht. Dies ist freilich nur möglich, wenn kurzfristige Strategien verfolgt werden und Alternativen unterlassen werden, die sich erst langfristig rechnen. Kapitalmärkte gewinnen also weltweit gegenüber traditioneller der Finanzierung durch Banken an Gewicht.

Der sich entwickelnde kapitalmarktgetriebene Kapitalismus ist weder sozial noch ökologisch verträglich. Mit den verschiedenen Dimensionen dieser ökologischen Unzuträglichkeit und den Versuchen der Etablierung eines Umweltregimes beschäftigen sich die Beiträge von Ulrich Brand/Christoph Görg und Cord Jakobeit.

Eine Re-Regulierung der Finanzmärkte, also eine gewisse Politisierung der globalen Ökonomie steht auf der Tagesordnung. Doch was ist davon zu erwarten? Von der Entschleunigung der Finanzflüsse, von der Einführung einer Haftung der Finanzanleger im Fall von Finanzkrisen, einer Beendigung der Auflagenpolitik des IWF, einem selektiven Einsatz von Kapitalverkehrskontrollen und von einer teilweisen Streichung der Schulden von Ländern der Dritten Welt (Vorschläge aus einem Diskussionspapiers für den Ratschlag von Nichtregierungsorganisationen zur Regulierung der internationalen Finanzmärkte im Januar 2000 in Frankfurt)? Wenn diese Maßnahmen tatsächlich das Risiko von globalen Finanzanlagen mindern, spricht vieles dafür, dass das Zinsniveau insgesamt sinken wird, was langfristige Anlagen in der Realwirtschaft möglicherweise wieder rentabler machen würde. Allerdings darf nicht zu viel erwartet werden. Sofern die Finanzkrisen ein Ausdruck der Funkti- 
onsweise der internationalen Finanzmärkte sind, lassen sie sich durch eine ReRegulierung der Finanzmärkte mit den diskutierten Maßnahmen eindämmen. Einen krisenfreien Kapitalismus darf man jedoch auch dann nicht erwarten, wenn alle Maßnahmen umgesetzt würden. Denn wenn auch möglicherweise die Realzinsen durch geeignete Regulierung gesenkt werden könnten, ist es kaum möglich, die realen Wachstumsraten des Sozialprodukts stark anzuheben. Auch wenn es heute selbst unter Grünen modisch ist, eine Steigerung des Wachstums für sinnvoll und machbar zu erklären, bleiben ökologische Schranken, und zwar sowohl auf der Ressourcenseite wie bei den Emissionen der Energie- und Stoffflüsse. Darüber hinaus aber gibt es auch ökonomische Schranken. Die marginale Kapitalproduktivität, also der Zuwachs des Sozialprodukts, der mit einer zusätzlichen Investition erzielt werden kann, ist in den vergangenen Jahrzehnten gesunken. Die Folge: Es müßten immer höhere Investitionsbeträge aufgewendet werden, um auch nur die bereits erreichte Wachstumsrate zu halten. Dies ist zeitweise in "guter" Konjunktur möglich, aber wohl kaum dauerhaft. Obendrein könnten die Investitionen wohl nur auf dem Wege der Umverteilung zu Gunsten der Kapitaleigner angehoben werden. Allerdings könnten sinkende Realzinsen für ein selektives Wachstum etwa für Investitionen in erneuerbare Energien genutzt werden, um einen Weg aus der fossilen Sackgasse zu bahnen. Hier müßten Energie- und Umweltpolitik, die Chancen, die eine Regulierung der Finanzmärkte bietet, ergreifen.

V.

Und mehr noch: In den Beiträgen von Heribert Dieter und Susanne Lütz wird gezeigt, dass die USA derzeit kein ausge- prägtes Interesse an einer Re-Regulierung der globalen Finanzmärkte haben. Dies ist zweifellos richtig, auch wenn Präsident Clinton auf dem Weltwirtschaftsforum von Davos im Januar 2000 Reformen der Weltwirtschaftsordnung angemahnt hat doch nur wenn sie in Richtung Liberalisierung und des Einbezugs jener Länder geht, die bislang noch nicht vollständig ins globale System integriert sind. Dies gilt vor allem für China. Die Finanzinstitutionen der USA nutzen die deregulierten Finanzmärkte für ihre Klientel von Geldvermögensbesitzern, um hohe Gewinne zu erzielen, auch wenn diese durch extreme Ausbeutung von einzelnen Schuldnern, ja von nationalen Gesellschaften, die extern verschuldet sind, zustande kommen. Die Verteilung der Einkommen und Vermögen in der Welt wird auf diese Weise zwar immer ungleicher. Doch damit können diejenigen, die über Geldvermögen verfügen, in "Gods own country“ gut und angenehm leben, zumal wenn man sich gegen die prognostizierbaren sozialen und politischen Konflikte oder die finanziellen und ökologischen Krisen mit überlegener militärischer Macht schützen kann. Die USA sind die ,einzige Weltmacht" (Brzezinski) und sie verhalten sich in der Schulden- und Finanzkrise (und selbst in der globalen Umweltpolitik) als ein „predatory hegemon“, als ein Land, das seine finanzielle, politische und militärische Macht einsetzt, um in einer Welt großer Gegensätze Vorteile für sich auf Kosten anderer Länder zu erzielen (vgl. dazu auch Jim O'Connor). Dies bedeutet: Die politische Klasse der USA, die die Karte des „unipolar moment" spielt, ist heute das größte Hindernis einer Neuordnung des globalen Finanzsystems. Eine Re-Regulierung der Finanzmärkte wird vielleicht in Gang gesetzt, aber die Krisenhaftigkeit des globalen Finanzsystems bleibt. 
Nach den Erfahrungen der schweren Finanzkrisen der 80er und 90er Jahre ist ReRegulierung zwar kein Un-Wort mehr. Um die Folgen der Finanzkrisen, vor allem um das „Systemrisiko“ zu vermeiden, werden Verhaltensregeln von Finanzinstitutionen, Regeln für die Funktionsweise von Märkten etc. erwogen und manchmal praktiziert. Dazu sind Akteure auf Finanzmärkten sehr schnell bereit, wie bei der Rettungsaktion des Long Term Capital Management Hedge-Funds im September 1998, der in wenigen Tagen mit Milliarden-Spritzen in einer Art ,publicprivate partnership“ zwischen Regierungen, Zentralbanken und privaten Banken, die in der globalen Liga „spielen“, vor der Pleite bewahrt wurde, weil diese möglicherweise viele Banken, und dann eben „das System“ mitgerissen hätte. Allerdings sind die Lehren der Fast-Pleite des LTCM oder der Asienkrise wenige Monate später fast vergessen (Le Monde, 13.10.1999) auch dies ein Ausdruck des ,short-terminism“ der globalen Finanzmärkte.

Angesichts der offensichtlichen Krisenhaftigkeit der globalen Finanzmärkte und der Schwierigkeit wirksamer Regulierung hat sich schon seit Jahren oder Jahrzehnten eine Form von „soft regulation“, von „informeller Politik“ auf den Weltmärkten herausgebildet. Neben den internationalen Institutionen von Währungs- und Finanzsystem, den großen Nationalstaaten und ihren „Clubs“ (G7, G10 etc.) treten auch quasi-öffentliche private Akteure auf den Plan der Reformdebatten, die sich ihrerseits global vernetzen. Dazu gehören große private Stiftungen mit globaler oder zumindest transnationaler Ausstrahlung (von der Heritage Foundation bis Bertelsmann) oder das World Economic Forum von Davos, das Jahr für Jahr die politische und ökonomische Elite der globalisierten Welt zusammenführt. Dort werden
Regeln sozusagen diskursiv „erlassen“ - im Doppelsinn des Wortes: ihr Erlass wird zwar vorgeschlagen, doch wird zugleich ihre Verbindlichkeit unterlassen. Aber indem sich die „business community“ und die politischen Führer darauf einlassen, erlangen sie tatsächliche Relevanz - freilich außerhalb jeder politisch-demokratischen Kontrolle durch einen Souverän. Im Gegenteil, aus dieser Welt der Geschäfte werden Einflüsse auf die Politik ausgeübt, die die Souveränität des Volkssouveräns in Frage stellen. Dies geschieht vor allem durch mächtige Lobby-Gruppen. Beispielsweise durch die „European Round Table of Industrialists“, von der „The Guardian“ (16.12.1999) schreibt, daß ihr Einfluß auf die Politik der EUKommission entscheidend sei - wichtiger als der Einfluß gewählter Volksvertreter im Europa-Parlament und selbst größer als der mächtiger Regierungen. Wie groß die Macht der Ökonomie über die Politik ist, erhellt schlaglichtartig auch der Korruptionsskandal, der das deutsche politische System zu Beginn des neuen Jahrhunderts erschüttert. Die Regulierung ist zwar weich in der Welt informeller Politik, doch die Einflussnahme außerkonstitutioneller ökonomischer Mächte ist hart und brutal.

International vernetzt sind aber nicht nur kapitalstarke pressure-groups, sondern auch Nichtregierungsorganisationen, Expertengruppen und lokale Bündnisse, die sich gegen die Negativfolgen der Globalisierung engagieren. Dass sich hier nicht nur verschiedene Einflußgruppen überlappen, sondern dass die Nationalstaaten gar nicht mehr isoliert, lediglich ihrem „eigenen“ Interesse folgend agieren können, wird von den Vertretern des „Global Governance“-Ansatzes geltend gemacht. In PROKLA 116 wurde dieser Ansatz von Achim Brunnengräber und Christian 
Stock kritisiert. Dirk Messner versucht nun in seinem Beitrag den Ertrag des Global Governance Ansatzees deutlich zu machen, Franz Nuscheler antwortet direkt auf verschiedene Kritiker. Im Kontext der Wandlungen der US-amerikanischen Außenpolitik wird der Global GovernanceAnsatz im Aufsatz von Christoph Scherrer verortet.

VI.

Eine isolierte (Re-)Regulierung der Finanzmärkte am Beginn des 21. Jhs. ist ebenso wirksam wie die „Organisierung des Kapitalismus“" es in der ersten Hälfte des 20. Jhs. gewesen ist. (Re-)Regulierungen innerhalb der gegenwärtigen Weltwährungsordnung mit unregulierten internationalen Kapitalmärkten, Währungskonkurrenz zwischen Dollar, Euro und Yen und flexiblen Wechselkursen zwischen den wichtigsten Währungen der Welt ändern nicht die Logik der Entwicklung und der Instabilität des Weltwährungs- und Finanzsystems. An dieser Stelle scheitert der Diskurs über Regulierung, denn nun müßte eine prinzipielle Ent- scheidung getroffen werden zwischen den Interessen derjenigen, die ihr Kapital verwerten wollen (und müssen) und den anderen, die Finanzbeziehungen einsetzen wollen, um Entwicklung, Arbeitsplätze, ökologische Projekte zu fördern. Das Ergebnis ist vorhersehbar. Die zweite Gruppe ist in den öffentlichen Diskursen der „Zivilgesellschaft“ präsent, doch die erste Gruppe setzt sich praktisch durch, weil sie unter der Fuchtel der Konkurrenz Rendite vorweisen muß und danach das politische Regelsystem gestaltet. Ein Ende der Finanzkrisen ist so lange nicht absehbar, wie es Finanzmärkte gibt.

Resignation also? Keineswegs. Die Warnung vor einer Überschätzung der Möglichkeiten einer Regulierung der Weltwirtschaft bedeutet nicht, daß das ganze Projekt sinnlos wäre. Man darf nur nicht zu viel erwarten. Wer mehr erwarten und erreichen will, muß an die Frage der Gestaltung der globalen ökonomischen und politischen Beziehungen allerdings mit größerer Radikalität herangehen, als sie in den Vorschlägen der Re-Regulierung enthalten ist.

\section{PROKLA 119 (Juni 2000): Chinesischer Kapitalismus}

Als einziges „großes“ Land bekennt sich China noch zum Sozialismus - und ist doch dabei, in immer schnellerem Tempo den Kapitalismus einzuführen. Nicht nur auf der ideologischen, sondern auch auf der ökonomischen, sozialen und politischen Ebene steckt dieser Prozeß voller Widersprüche: der steuernde Staat kollidiert mit der von ihm entfesselten Privatwirtschaft, der Partei bricht die Basis weg, während die Spitzen in Korruption versinken, die Differenzen zwischen Stadt und Land, zwischen verschiedenen Regionen und Bevölkerungsschichten nehmen immer stärker zu. Die Gefahr einer tiefgreifenden Transformationskrise steigt, während sich gleichzeitig Anzeichen eines verstärkten Nationalismus erkennen lassen. Entwicklungen, die nicht nur für China selbst, sondern für ganz Asien unmittelbare Auswirkungen haben werden. 